\title{
EXTRAÇÃO DE CORANTE TÊXTIL EM SISTEMAS AQUOSOS BIFÁSICOS BASEADOS EM LÍQUIDOS IÔNICOS
}

\author{
R. C. S. SOUSA ${ }^{1}$, P. S. TRINDADE ${ }^{1}$, B. A. F. TEIXEIRA ${ }^{1}$, O. L. FLAUSINO ${ }^{1}$, M. T. NEVES ${ }^{1}$ e \\ C. R. BELLATO ${ }^{1}$ \\ ${ }^{1}$ Universidade Federal de Viçosa, Departamento de Química e Engenharia Química \\ E-mail para contato: rita.sousa@ufv.br
}

\begin{abstract}
RESUMO - Os líquidos iônicos (LI) aplicados em sistemas aquosos bifásicos (SAB) têm sido recentemente usados em processos de extração de corantes. O objetivo desse trabalho foi estudar a partição do corante vermelho congo, usando SAB formados pelos $\mathrm{LI}\left(\mathrm{C}_{4} \mathrm{minCH}_{3} \mathrm{SO}_{3}\right.$ e $\left.\mathrm{C}_{4} \mathrm{minCl}\right)$, fosfato de potássio e água para diferentes concentrações de $\mathrm{LI}$. Os $\mathrm{SAB}$ foram preparados pela pesagem adequada dos componentes, de acordo com dados de equilíbrio disponíveis em literatura. Após, os tubos foram centrifugados e deixados em banho termostático a $298 \mathrm{~K}$ por 8 horas. A quantificação do corante nas fases formadas após equilíbrio foi feita por espectofotometria. $\mathrm{O}$ coeficiente de partição foi determinado pela razão das concentrações de corante nas fases superior (rica em LI) e inferior (rica em sal). Os seus valores variaram de 1,69 a 1,76 para o $\mathrm{SAB}$ formado pelo $\mathrm{C}_{4} \mathrm{minCl}$, e foi encontrado o valor de 1,889 para o $\mathrm{C}_{4} \operatorname{minCH}_{3} \mathrm{SO}_{3}$. Foi também determinada a $\%$ de recuperação teórica, sendo os maiores valores encontrados para $\mathrm{C}_{4} \mathrm{minCH}_{3} \mathrm{SO}_{3}$, com valor próximo de $50 \%$. Os resultados obtidos indicam o uso de SAB baseados em LI um método eficiente para ser empregado na extração de corantes.
\end{abstract}

\section{INTRODUÇÃO}

A descarga de efluentes aquosos contendo elevada quantidade de corantes pelas indústrias têxteis conduz a preocupações ambientais e econômicas. O uso extensivo de corantes leva a diversos problemas para a saúde humana e para o ecossistema. A maioria dos corantes são substâncias cancerígenas, mutagênicas, alergênicas e tóxicas por natureza. Além disso, a presença desses corantes é facilmente detectável em água, mesmo em uma concentração muito baixa. Sendo assim, sua presença é bastante indesejável, uma vez que a percepção pública da qualidade da água é diretamente influenciada pela sua cor (Gharehbaghi e Shemirani, 2012). Portanto, novas leis ambientais estão sendo implementadas, e a remoção de corantes têxteis de efluentes tem sido um assunto de grande interesse nos últimos anos (Sanghi e Bhattacharya, 2002; Malik, 2003).

Como uma nova e eficiente abordagem para remover corantes em efluentes aquosos, o processo de extração líquido-líquido utilizando líquidos iônicos (LI) aplicados em sistemas aquosos bifásicos (SAB) tem despertado grande interesse industrial e acadêmico (Vijayaraghavan, 2006).

A grande vantagem do uso de LI como uma alternativa a outros solventes em SAB consiste na habilidade de dissolver uma variedade de compostos orgânicos e iônicos em quantidade apreciável. Na maioria dos casos imiscíveis em água, os LI promovem uma extração seletiva de 
compostos de uma fase aquosa, permitindo a pré-concentração e separação de produtos de interesse.

Os LI são sais compostos por íons grandes que não formam uma rede cristalina bem definida e, assim, permanecem líquidos à temperatura ambiente ou perto desta. Estes compostos são solventes interessantes, com características "verdes", com pressão de vapor negligenciável mesmo em elevadas temperaturas e oferecem diversas vantagens como a recuperação dos produtos, reciclabilidade, não-inflamabilidade em condições ambiente, estabilidade térmica e química elevada e grande capacidade de solvatação para ampla variedade de compostos tais como espécies orgânicas ou íons metálicos (Han e Armstrong, 2007).

Assim, o propósito deste projeto é realizar um estudo sistemático envolvendo diferentes tipos de LI a fim de se obter novas informações sobre a sua capacidade de extração de corantes de indústrias têxteis. Os SAB a serem estudados serão compostos por diferentes tipos de LI para avaliar a influência das características estruturais do LI e da sua concentração na eficiência de extração de vermelho congo através da determinação dos coeficientes de partição em cada condição experimental.

\subsection{Corantes têxteis}

Os efluentes oriundos de processo industrial têxtil envolvendo etapas de tingimento com corantes são altamente poluentes, de modo que grandes quantidades destes despejos acarretam grandes problemas ambientais. Além da interferência estética, quando não tratados adequadamente e lançados em águas naturais, os efluentes provenientes da indústria de corantes ou de processos envolvendo tingimento de fibras têxteis podem interferir na absorção da luz pelos vegetais e animais do ambiente aquático, provocando modificações nas atividades fotossintetizantes da biota aquática (Zanoni e Carneiro, 2001). Os corantes são empregados nas mais diversificadas indústrias, contando com um alto consumo anual. Seus efeitos são maléficos, já que são substâncias carcinogênicas. A maioria dos corantes comercialmente usados é resistente à biodegradação, à fotodegradação e à ação de agentes oxidantes. Diante de compostos corantes de difícil degradação contidos nos efluentes têxteis, existem inúmeras pesquisas de métodos inovadores visando a redução da poluição ambiental.

\subsection{Sistemas aquosos bifásicos baseados em líquidos iônicos}

A extração líquido-líquido por sistemas aquosos bifásicos (SAB) é uma operação muito utilizada na indústria química como técnica de separação. Os SAB são formados por espécies químicas que, quando misturadas em determinadas faixas de composição e temperatura, dividemse em duas fases com composições diferentes, em equilíbrio termodinâmico. A purificação é resultado de uma partição diferenciada da molécula-alvo e impurezas entre as duas fases líquidas (Pessoa Jr. e Kilikian, 2005). Solutos se distribuem entre as duas fases, dependendo da sua afinidade relativa por cada uma das fases individuais (Albertsson, 1986). Por isto, a predição e a interpretação da partição de corantes em SAB demandam muitos estudos. 
A extração líquido-líquido é uma tecnologia convencional de controle de contaminantes no ambiente. O projeto de um solvente seguro e ambientalmente benigno desempenha um papel cada vez mais importante no desenvolvimento desta tecnologia de extração. Alguns líquidos iônicos são solventes de extração adequados para poluentes orgânicos, devido à sua imiscibilidade com água, bem como a sua elevada solubilidade das espécies orgânicas.

Os LI são sais líquidos a temperatura ambiente e são normalmente formados por um cátion orgânico relativamente grande e um ânion orgânico ou inorgânico relativamente pequeno. Estas substâncias diferem dos sais comuns por apresentarem uma fraca interação interiônica, a qual resulta em uma baixa energia do retículo cristalino e baixa temperatura de fusão (Cassol, 2007). O estudo dos líquidos iônicos estáveis começou na década de 60, mas nos últimos dez anos tem havido um crescimento exponencial de publicações relacionadas a eles.

Os LI possuem propriedades físico-químicas muito interessantes, destacando-se as seguintes características: são usualmente líquidos em uma ampla faixa de temperatura próxima à temperatura ambiente; apresentam viscosidade relativamente baixa; não são inflamáveis; apresentam estabilidade térmica e eletroquímica maiores que solventes orgânicos clássicos; dissolvem inúmeros compostos orgânicos e inorgânicos; podem ser modulados pela seleção do cátion ou ânion; são estáveis ao ar; são facilmente recicláveis; são sintetizados a partir de reagentes comerciais acessíveis e utilizando métodos sintéticos de relativa simplicidade (Cassol, 2007).

A extração em SAB utilizando LI é uma nova técnica de separação de compostos. Estes LI são empregados na formação de SAB quando um sal inorgânico, como o fosfato de potássio, é usado como um segundo componente na formação das fases. Acima de concentrações críticas estes compostos em solução aquosas promovem a separação de fases resultando na formação de uma fase rica em LI (superior) e outra enriquecida em sal (inferior) (Liu et al., 2011).

Algumas literaturas mostraram que a extração líquido-líquido utilizando LI para remoção de corantes é uma tecnologia eficaz (Vijayaraghavan et al., 2006;. Li et al, 2007; Pei et al., 2007). Os resultados obtidos até o presente momento são muito promissores e indicam um grande potencial de aplicação. Porém, de acordo com nosso conhecimento, pouca ou nenhuma informação tem sido reportada em literatura a respeito de comportamento de partição de vermelho congo nos LI a serem estudados neste trabalho. Tais informações serão muito importantes para design de "processos verdes" de purificação dos mesmos.

\section{MATERIAL E MÉTODOS}

Os líquidos iônicos utilizados neste trabalho ([ $\left.\mathrm{C}_{4} \mathrm{mimCl}\right]$, 1-butyl-3-methylimidazolium chloride e $\left[\mathrm{C}_{4} \mathrm{minCH}_{3} \mathrm{SO}_{3}\right.$ ], 1-butyl-3-methylimidazolium methanesulfonate) foram adquiridos da Sigma Aldrich. Os sais inorgânicos foram adquiridos da Merck Brasil com pureza maior que $98 \%$ $(\mathrm{m} / \mathrm{m})$. Todos os reagentes utilizados foram de grau analítico.

\subsection{Preparo dos sistemas aquosos bifásicos baseados em líquidos iônicos}

Os $\mathrm{SAB}$ foram preparados de acordo com dados de equilíbrio encontrados em literatura 
(Ventura et al., 2011), de modo a obter sistemas com três diferentes concentrações em massa de $\mathrm{C}_{4} \operatorname{minCl}(15,75 \%, 17,69 \%$ e $21,36 \%)$ e duas concentrações de $\mathrm{C}_{4} \mathrm{minCH}_{3} \mathrm{SO}_{3}(25,03 \%$ e 30,33\%). As massas dos componentes adicionados ao SAB são apresentadas na Tabela 1 e na Tabela 2, para $\mathrm{C}_{4} \operatorname{minCl}$ e $\mathrm{C}_{4} \mathrm{minCH}_{3} \mathrm{SO}_{3}$, respectivamente.

Tabela 1 - Composição mássica dos SAB para diferentes valores de concentração do LI $\mathrm{C}_{4} \mathrm{minCl}$

\begin{tabular}{|c|c|c|c|}
\hline & $15,75 \%$ LI & $17,69 \%$ LI & $21,36 \% \mathrm{LI}$ \\
\hline $\mathrm{m}_{\text {sal }}(\mathrm{g})$ & 2,2667 & 2,1537 & 2,3037 \\
\hline $\mathrm{m}_{\text {água }}(\mathrm{g})$ & 6,0583 & 5,9773 & 5,4603 \\
\hline $\mathrm{m}_{\text {LI }}(\mathrm{g})$ & 1,5750 & 1,7690 & 2,1360 \\
\hline
\end{tabular}

Tabela 2 - Composição mássica dos SAB para diferentes valores de concentração do LI $\mathrm{C}_{4} \mathrm{minCH}_{3} \mathrm{SO}_{3}$

\begin{tabular}{|c|c|c|}
\hline & $25,03 \% \mathrm{LI}$ & $30,33 \% \mathrm{LI}$ \\
\hline $\mathrm{m}_{\text {sal }}(\mathrm{g})$ & 1,4964 & 1,4857 \\
\hline $\mathrm{m}_{\text {água }}(\mathrm{g})$ & 5,9006 & 5,3813 \\
\hline $\mathrm{m}_{\mathrm{LI}}(\mathrm{g})$ & 2,5030 & 3,0330 \\
\hline
\end{tabular}

As Tabelas 1 e 2 mostram a quantidade (\% em massa) adicionada de cada componente do sistema, para formar um SAB de massa total 10 gramas. As concentrações em massa de LI utilizadas foram escolhidas de modo que houvesse formação de fases.

A solução do corante foi adicionada, em $0,1 \mathrm{~mL}$ da solução com concentração pré-definida de 50 ppm, a tubos de centrífuga graduados contendo SAB formados pelo sal inorgânico e o LI. A mistura foi agitada vigorosamente e, então, os tubos foram centrifugados (Centrífuga FANEM Excelsa II 206 BL) a 2500 rpm por 5 min para completa separação de fases. Logo após, os tubos foram colocados em banho termostático ( Estufa FANEM, 206), com temperatura regulada a 298 $\mathrm{K}$ até se atingir o equilíbrio de fases. Os volumes de cada fase foram registrados. Amostras da fase superior rica em LI e da inferior, rica em sal, serão coletadas com seringas de $5 \mathrm{~mL}$ e diluídas para determinação da concentração de corante.

A concentração do corante nas fases foi determinada pelo método espectrofotométrico (Espectofotômetro Agilent, 8453 ) no comprimento de onda $598 \mathrm{~nm}$. A concentração do corante na fase superior, rica em LI, foi determinada por meio de uma curva analítica previamente estabelecida, Equação 1 para o $\mathrm{C}_{4} \mathrm{minCl}$ e Equação 2 para o $\mathrm{C}_{4} \mathrm{minCH}_{3} \mathrm{SO}_{3}$. Igualmente, a concentração da fase inferior, rica em sal, pode ser determinada pela curva analítica expressa pela Equação 3.

$$
\begin{aligned}
& C_{\text {sup }, 1}=1,998 \cdot 10^{-5} \cdot a b s+3,001 \cdot 10^{-7} \\
& C_{\text {sup }, 2}=2,0 \cdot 10^{-5} \cdot a b s+3,102 \cdot 10^{-7} \\
& C_{\text {inf }}=2,0 \cdot 10^{-5} \cdot a b s+3,00 \cdot 10^{-6}
\end{aligned}
$$


Sendo abs, as absorvâncias do corante aferidas em cada fase dos SAB analisados.

Para eliminar a influência da presença do LI na análise da concentração de corante, um sistema controle foi preparado nas mesmas condições, mas sem adição de corante. O coeficiente de partição foi obtido pela Equação 4.

$$
K=C_{\text {sup }} / C_{\text {in }}
$$

Em que $\mathrm{C}_{\text {sup }}$ e $\mathrm{C}_{\text {inf }}$ correspondem às concentrações de equilíbrio do corante na fase superior (rica em LI) e na fase inferior (rica em fosfato), respectivamente.

Com o objetivo selecionar os SAB com melhor capacidade de purificação de corantes têxteis, foi calculada uma recuperação teórica (Y\%) na fase superior por meio da Equação 5:

$$
Y(\%)=100 /(1+(K / R))
$$

Sendo $\mathrm{R}$ a razão entre o volume da fase superior e inferior do sistema SAB em estudo.

\section{RESULTADOS E DISCUSSÃO}

As Tabelas 3 e 4 mostram os coeficientes de partição teóricas obtidos para vermelho congo em $\mathrm{C}_{4} \mathrm{minCl}$ e $\mathrm{C}_{4} \mathrm{minCH}_{3} \mathrm{SO}_{3}$, respectivamente.

Tabela 3 - Partição de vermelho congo em $\mathrm{C}_{4} \mathrm{minCl}$

\begin{tabular}{|c|c|c|c|c|c|}
\hline \multirow{2}{*}{$\begin{array}{c}\text { Concentração } \\
\text { LI(\%) }\end{array}$} & \multicolumn{2}{|c|}{ Concentração de corante } & \multicolumn{2}{c|}{ Volume da fase (mL) } & \multirow{2}{*}{$\begin{array}{c}\text { Coeficiente de } \\
\text { partição (K) }\end{array}$} \\
\cline { 2 - 5 } & Fase Superior & Fase Inferior & Superior & Inferior & - \\
\hline 15,75 & - & - & - & - & 1,7629 \\
\hline 17,69 & 0,005988 & 0,003125 & 4,600 & 5,000 & 1,6935 \\
\hline 21,36 & 0,005630 & 0,003290 & 4,775 & 4,825 & \\
\hline
\end{tabular}

Tabela 4 - Partição de vermelho congo em $\mathrm{C}_{4} \operatorname{minCH}_{3} \mathrm{SO}_{3}$

\begin{tabular}{|c|c|c|c|c|c|}
\hline \multirow{2}{*}{$\begin{array}{c}\text { Concentração } \\
\text { LI(\%) }\end{array}$} & \multicolumn{2}{|c|}{ Concentração de corante } & \multicolumn{2}{c|}{ Volume da fase (mL) } & Coeficiente de \\
\cline { 2 - 5 } & Fase Superior & Fase Inferior & Superior & Inferior & partição (K) \\
\hline 25,03 & - & - & - & - & - \\
\hline 30,33 & 0,003898 & 0,003943 & 6,5 & 3,4 & 1,8898 \\
\hline
\end{tabular}

Os resultados apresentados nas Tabelas 3 e 4 mostram que em alguns sistemas $\mathrm{SAB}$ não ocorreu separação de fases, em $15,75 \%$, para o $\mathrm{C}_{4} \operatorname{minCl}$, e em $25,03 \%$ para o $\mathrm{C}_{4} \operatorname{minCH}_{3} \mathrm{SO}_{3}$. É relevante mencionar que em algumas repetições realizadas para essas concentrações houve a formação de fases. Dessa forma, não se observa acurácia nos resultados relativos a essas composições do SAB. Tal efeito pode ocorrer devido à diferença de composição entre as fases determinada pelo parâmetro termodinâmico comprimento de linha de amarração (Pawaskar et al., 
1999) Quanto maior for esse comprimento, maior será a diferença entre as fases, diminuindo, portanto, a eficiência da extração do corante vermelho congo no SAB.

Os coeficientes de partição obtidos mostram que a extração do corante vermelho congo em diferentes LI foi eficiente, pois os valores apresentados foram maiores que 1, indicando que o corante foi concentrado na fase superior dos SAB estudados. Não houve diferenças significativas entre os líquidos iônicos quanto à distribuição do corante nas fases. $\mathrm{O}$ autor Vijayaraghavan (2006) estudando a partição de corantes ácido-azul e ácido-vermelho em SAB compostos por $N$ butyl, $N$-methyl pyrrolidinium bis(trifluoromethanesulfonyl) amide [p14][tf2N], água e sal inorgânico di sódico, obteve coeficientes de partição em torno de 2, semelhantes aos valores encontrados nesse estudo.

A Tabela 5 mostra os valores de recuperação teórica (Y\%) estimadas para os líquidos iônicos $\mathrm{C}_{4} \operatorname{minCl}$ e $\mathrm{C}_{4} \operatorname{minCH}_{3} \mathrm{SO}_{3}$.

Tabela 5- Recuperação Teórica(Y\%) do vermelho congo em $\mathrm{C}_{4}$ minCl e $\mathrm{C}_{4} \mathrm{minCH}_{3} \mathrm{SO}_{3}$

\begin{tabular}{|c|c|c|}
\hline Líquido Iônico & $\% \mathrm{LI}(\mathrm{m} / \mathrm{m})$ & Recuperação Teórica (Y\%) \\
\hline \multirow{3}{*}{$\mathrm{C}_{4} \mathrm{minCl}$} & 15,75 & - \\
\cline { 2 - 3 } & 17,69 & 34,29 \\
\cline { 2 - 3 } & 21,36 & 36,88 \\
\hline \multirow{2}{*}{$\mathrm{C}_{4} \mathrm{minCH}_{3} \mathrm{SO}_{3}$} & 25,03 & - \\
\cline { 2 - 3 } & 30,33 & 50,28 \\
\hline
\end{tabular}

Os resultados estimados para a recuperação teórica indicam que o liquido iônico $\mathrm{C}_{4} \operatorname{minCH}_{3} \mathrm{SO}_{3}$ é mais eficiente no processo de purificação do corante vermelho congo, apresentando valores de recuperação teórica próximos de $50 \%$. Não foi possível analisar a influência da concentração do líquido iônico no resultado da recuperação teórica pois ainda não foi possível obter mais dados de equilíbrio para os SAB estudados.

\section{CONCLUSÃO}

A extração de efluentes oriundos de processos industriais têxteis é uma área emergente, porém apresenta estudos promissores para a redução dos efeitos poluentes e nocivos desses compostos.

Neste trabalho estudou-se a partição do corante têxtil vermelho congo em SAB constituídos por dois tipos de líquidos iônicos e fase aquosa salina. Os resultados apresentados mostraram que o SAB com base em LI é um método de extração eficiente, pois os líquidos iônicos apresentam características físico químicas importantes para a formação de fases, e também são uma alternativa aos solventes orgânicos utilizados convencionalmente em métodos de extração.

Apesar do progresso alcançado mediante o aumento no estudo da partição de íons e biomoléculas em SAB, ainda são necessários estudos que forneçam mais dados de equilíbrios de partição para diversos LI e suas fases aquosas, e ainda novas investigações que contribuam para a compreensão dos parâmetros que explicam a partição de corantes têxteis nos SAB. Dessa forma, o potencial extrator em escala industrial pode ser otimizado, assim como sua aplicabilidade nas indústrias que envolvem etapas de tingimento. 


\section{REFERÊNCIAS}

ALBERTSSON, P. A. Partition of cell and macromolecules. New York: John Wiley, 346 p. 1986.

CASSOL, C. C. Líquidos iônicos em processos de extração seletiva de compostos aromáticos, nitrogenados e sulfurados em frações do petróleo. Dissertação de Mestrado, Universidade Federal do Rio Grande do Sul, Instituto de Química, 2007.

GHAREHBAGHI, M.; SHEMIRANI, F. A Novel method for dye removal: ionic liquid-based dispersive liquid-liquid extraction (IL-DLLE). Research Article, Clean - Soil, Air, Water v. 40 (3), p. 290-297, 2012.

HAN, X. ARMSTRONG, D.W. Ionic liquids in separations, Acc. Chem. Res. v. 40, p. 1079-1086. 2007.

LI, C. P. XIN, B. P. XU, W. G. ZHANG, Q. Study on the extraction of dyes into a roomtemperature ionic liquid and their mechanisms, J. Chem. Technol. Biotechnol. v. 82 (2), p. 196, 2007.

LIU, Y. YU, Y.L. CHEN M.Z. XIAO. X. Advances in aqueous two-phase systems and applications in protein separation and purification. Canadian Journal on Chemical Engineering and Technology, v. 2, 2011.

MALIK, P. K. Use of activated carbons prepared from sawdust and rice-husk for adsorption of acid dyes: a case study of acid yellow 36, Dyes Pigm. 56 (3), 239. 2003.

PAWASKAR, C. S.; MOHAPATRA, P. K.; MANCHANDA, V. K.; Extraction of actinidesand fission products from salt solutions using polyethylene glycols (PEGs). J. Radioanal. Nucl. Chem. v. 242, p. 627-634., 1999

PEI, Y. C.; WANG, J. J.; XUAN, J X.; FAN, P.; FAN, M. H. Factors affecting ionic liquids based removal of anionic dyes from water, Environ. Sci. Technol. v. 41 (14), p. 5090, 2007.

PESSOA, Jr. e KILIKIAN, B. V. Purificação de Produtos Biotecnológicos. Manole, Barueri, SP, 2005.

SANGHI, R.; BHATTACHARYA, B. Review on decolorisation of aqueous dye solutions by low cost adsorbents, Color. Technol. v. 118 (5), p. 256, 2002.

VENTURA, S. P. M.; SOUSA, S. G.; FREIRE, M. G.; LIMA, A. S.; COUTINHO, J. A. P. Design of ionic liquids for lipase purification. J. Chromatogr., B v. 879, p. 2679-2687, 2011.

VENTURA, S.P.M.; NEVES, C.; FREIRE, M.G.; MARRUCHO, I.M.; OLIVEIRA, J.; COUTINHO, J.A.P. Evaluation of anion influence on the formation and extraction capacity of ionic-liquid-based aqueous biphasic systems. Journal of Physical Chemistry B, v. 113(27), p. 9304-9310, 2009.

VIJAYARAGHAVAN, R. VEDARAMAN, N. SURIANARAYANAN, M. MacFARLANE, D. R. Extraction and recovery of azo dyes into an ionic liquid, Talanta, v. 69 (5), p. 1059, 2006. 
ZANONI, M. V.; CARNEIRO, P. A. O descarte dos corantes têxteis. Ciência Hoje, v. 29, p. 6164, 2001. 